\title{
Slide Attacks
}

\author{
Alex Biryukov and David Wagner ${ }^{\star \star}$
}

\begin{abstract}
It is a general belief among the designers of block-ciphers that even a relatively weak cipher may become very strong if its number of rounds is made very large. In this paper we describe a new generic known- (or sometimes chosen-) plaintext attack on product ciphers, which we call the slide attack and which in many cases is independent of the number of rounds of a cipher. We illustrate the power of this new tool by giving practical attacks on several recently designed ciphers: TREYFER, WAKE-ROFB, and variants of DES and Blowfish.
\end{abstract}

\section{Introduction}

As the speed of computers grows, fast block ciphers tend to use more and more rounds, rendering all currently known cryptanalytic techniques useless. This is mainly due to the fact that such popular tools as differential [1] and linear analysis [13] are statistic attacks that excel in pushing statistical irregularities and biases through surprisingly many rounds of a cipher. However any such approach finally reaches its limits, since each additional round requires an exponential effort from the attacker.

This tendency towards a higher number of rounds can be illustrated if one looks at the candidates submitted to the AES contest. Even though one of the main criteria of the AES was speed, several prospective candidates (and not the slowest ones) have really large numbers of rounds: RC6(20), MARS(32), SERPENT(32), CAST(48). This tendency is a reflection of a belief/empirical evidence that after some high number of rounds even a relatively weak cipher becomes very strong. It is supported by the example of DES, where breaking even 16 rounds is already a very hard task, to say nothing about 32-48 rounds (e.g. double- or triple-DES). Thus for the cryptanalyst it becomes natural to search for new tools which are essentially independent of the number of rounds of a cipher. The first step in this direction can be dated back to a 1978 paper by Grossman and Tuckerman 9, which has shown how to break a weakened Feistel cipher 1 by a chosen plaintext attack, independent of the number of rounds. We were also inspired by Biham's work on related-key cryptanalysis [2], and Knudsen's early work [12].

* Applied Mathematics Department, Technion - Israel Institute of Technology, Haifa, Israel 32000. Email: albi@cs.technion.ac.il

** University of California, Berkeley. Email: daw@cs. berkeley.edu

${ }^{1}$ An 8-round Feistel cipher with eight bits of key material per round used to swap between two S-boxes $S_{0}$ and $S_{1}$ in a Lucifer-like manner. A really weak cipher by modern criteria. 
In this paper we introduce a new class of generic attacks which we call slide attacks together with a new set of cryptanalytic tools applicable to all product (mainly iterative) ciphers and even to any iterative (or recursive) process over the finite domain (stream ciphers, etc.). Such attacks apply as soon as the iterative process exhibits some degree of self-similarity and are in many cases independent of the exact properties of the iterated round function and of the number of rounds.

While the two other generic cryptanalytic attacks - differential and linear analysis - concentrate mainly on the propagation properties of the encryption engine (assuming a strong key-scheduling which produces independent subkeys), the degree of self-similarity of a cipher as studied by slide attacks is a totally different aspect. Depending on the cipher's design, slide attacks range from exploiting key-scheduling weaknesses to exploiting more general structural properties of a cipher. The most obvious version of this attack is usually easy to prevent by destroying the self-similarity of an iterative process, for example by adding iteration counters or fixed random constants. However more sophisticated variants of this technique are harder to analyze and to defend against.

We start by analyzing several block ciphers that decompose into $r$ iterations of a single key-dependent permutation $F_{i}$. We call such ciphers homogeneous. This usually arises when the key-schedule produces a periodic subkey sequence, when $F_{i}=F_{j}$ for all $i \equiv j \bmod p$ where $p$ represents the period. In the simplest case, $p=1$ and all round subkeys are the same. We call these attacks self-related key attacks, since they are essentially a special case of related-key attacks [2]. Note, however, that our attacks require only a known- (or sometimes chosen-) plaintext assumption and thus are much more practical than most related key attack 2 . For the case of block ciphers operating on a $n$-bit block, the complexity of slide attacks (if they work) is usually close to $O\left(2^{n / 2}\right)$ known plaintexts. For Feistel ciphers where the round function $F_{j}$ modifies only half of the block, there is also a chosen-plaintext variant which can often cut the complexity down to $O\left(2^{n / 4}\right)$ chosen texts.

A somewhat less expected observation is that schemes relying on key-dependent S-boxes are also vulnerable to sliding. In general, autokey ciphers and datadependent transformations are potentially vulnerable to such attacks. We summarize our results in Table 1 .

This paper is organized as follows. In Section 2, we describe the details of a typical slide attack, and in Section 3 we show how the attacks can be optimized for Feistel ciphers. We then proceed with an introductory example: a 96-bit DES variant with 64-rounds, which we call $2 \mathrm{~K}$-DES, Section 4 . The next two sections are devoted to cryptanalysis of several concrete cipher proposals: Section 5 breaks TREYFER, a cipher published in FSE'97, and Section 6 analyzes stream cipher proposals based on WAKE presented at FSE'97 and FSE'98. Finally, Section 7 shows slide attacks on ciphers with key-dependent S-boxes, focusing on a variant of Blowfish with zero round subkeys.

\footnotetext{
${ }^{2}$ However, Knudsen's early work on LOKI91 [12] showed how to use a related-key-type weakness to reduce the cost of exhaustive keysearch using only chosen plaintexts.
} 
Table 1. Summary of our attacks on various ciphers.

\begin{tabular}{lcrcc}
\hline Cipher & (Rounds) Key Bits & \multicolumn{2}{c}{ Our Attack } \\
\cline { 3 - 5 } & & \multicolumn{3}{c}{ Data Complexity Time Complexity } \\
\hline Blowfish $^{1}$ & $(16)$ & 448 & $2^{27} \mathrm{CP}$ & $2^{27}$ \\
Treyfer & $(32)$ & 64 & $2^{32} \mathrm{KP}$ & $2^{44}$ \\
2K-DES & $(64)$ & 96 & $2^{33} \mathrm{ACP}$ & $2^{33}$ \\
2K-DES & $(64)$ & 96 & $2^{32} \mathrm{KP}$ & $2^{50}$ \\
WAKE-ROFB & $(k)$ & $32 n$ & $2^{32} \mathrm{CR}$ & $2^{32}$ \\
\hline
\end{tabular}

1 - Modified variant, without round subkeys. KP - known-plaintext, CP - chosenplaintext, ACP - adaptive chosen-plaintext, CR - chosen-resynchronization (IV).

\section{A Typical Slide Attack}

In Figure 1, we show the process of encrypting the $n$-bit plaintext $X_{0}$ under a typical product cipher to obtain the ciphertext $X_{r}$. Here $X_{j}$ denotes the intermediate value of the block after $j$ rounds of encryption, so that $X_{j}=F_{j}\left(X_{j-1}, k_{j}\right)$. For the sake of clarity, we often omit $k$ by writing $F(x)$ or $F_{i}(x)$ instead of $F(x, k)$ or $F_{i}(x, k)$.

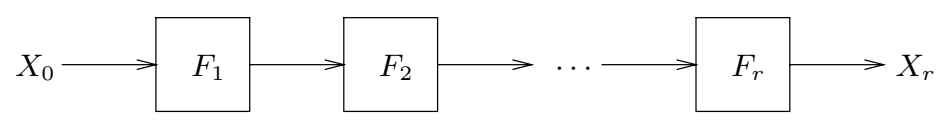

Fig. 1. A typical block cipher

As we mentioned before, the attack presented in this note is independent of the number of rounds of the cipher, since it views a cipher as a product of identical permutations $F(x, k)$, where $k$ is a fixed secret key (here $F$ might include more than one round of the cipher). Moreover its dependence on the particular structure of $F$ is marginal. The only requirement on $F$ is that it is very weak against known-plaintext attack with two plaintext-ciphertext pairs. More specifically, we call $F$ a weak permutation if given the two equations $F\left(x_{1}, k\right)=y_{1}$ and $F\left(x_{2}, k\right)=y_{2}$ it is "easy" to extract the key $k$. This is informal definition since the amount of easiness may vary from cipher to cipher. We can show that 3 rounds of DES form a weak permutation 3 . One and a half round IDEA is also weak.

\footnotetext{
${ }^{3}$ For $F=$ three rounds of DES, with DES keyschedule one may consider 4-bit output of specific S-box at the 1st and 3rd rounds. This gives a 4-bit condition on the 6 key bits entering this S-box at the 1st and on 6 bits entering this S-box at the 3rd round. Using similar observations it is possible to extract the full DES 56-bit key in time faster than that of one DES encryption.
} 
We next show in Figure 2 how a slide attack against such a cipher might proceed. The idea is to "slide" one copy of the encryption process against another copy of the encryption process, so that the two processes are one round out of phase. We let $X_{0}$ and $X_{0}^{\prime}$ denote the two plaintexts, with $X_{j}=F_{j}\left(X_{j-1}\right)$ and $X_{j}^{\prime}=F_{j}\left(X_{j-1}^{\prime}\right)$. With this notation, we line up $X_{1}$ next to $X_{0}^{\prime}$, and $X_{j+1}$ next to $X_{j}^{\prime}$.

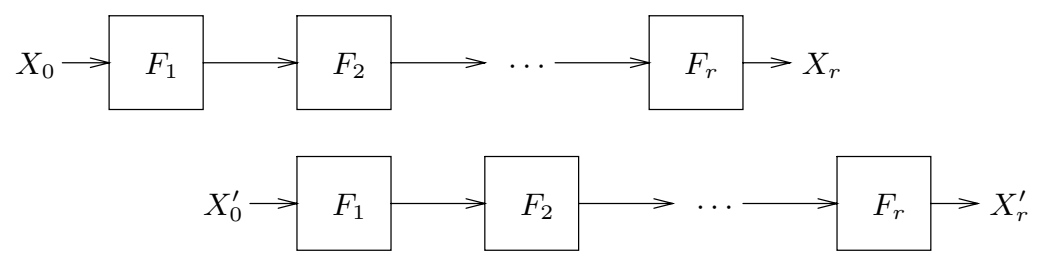

Fig. 2. A typical slide attack

Next, we suppose that $F_{j}=F_{j+1}$ for all $j \geq 1$; this is the assumption required to make the slide attack work. In this case, all the round functions are the same, so for the remainder of this section we will drop the subscripts and simply write $F$ for the generic round transform.

The crucial observation is that if we have a match $X_{1}=X_{0}^{\prime}$, then we will also have $X_{r}=X_{r-1}^{\prime}$. The proof is by induction. Suppose that $X_{j}=X_{j-1}^{\prime}$. Then we may compute $X_{j+1}=F\left(X_{j}\right)=F\left(X_{j-1}^{\prime}\right)=F\left(X_{j-1}^{\prime}\right)=X_{j}^{\prime}$, which completes the proof. Therefore, we call a pair $(P, C),\left(P^{\prime}, C^{\prime}\right)$ of known plaintexts (with corresponding ciphertexts) a slid pair if $F(P)=P^{\prime}$ and $F(C)=C^{\prime}$.

With this observation in hand, the attack proceeds as follows. We obtain $2^{n / 2}$ known texts $\left(P_{i}, C_{i}\right)$, and we look for slid pairs. By the birthday paradox, we expect to find about one pair of indices $i, i^{\prime}$ where $F\left(P_{i}\right)=P_{i^{\prime}}$, which gives us a slid pair.

Furthermore, slid pairs can often be recognized relatively easily. In general, we recognize slid pairs by checking whether it is possible that $F\left(P_{i}\right)=P_{i^{\prime}}$ and $F\left(C_{i}\right)=C_{i^{\prime}}$ both hold for some key. When the round function is weak, we are assured that this condition will be easy to recognize. Once we have found a slid pair, we expect to be able to recover some key bits of the cipher. If the round function is weak, we can in fact recover the entire key with not too much work. In general, we expect a single slid pair to disclose about $n$ bits of key material; when the cipher's key length is longer than $n$ bits, we may use exhaustive search to recover the remainder of the key, or we may alternatively obtain a few more slid pairs and use them to learn the rest of the key material.

Let us summarize the attack. For a cipher with $n$-bit blocks and repeating round subkeys, we need about $O\left(2^{n / 2}\right)$ known plaintexts to recover the unknown key. While a naive approach will require $O\left(2^{n}\right)$ work, much faster attacks are 
usually possible by exploiting the weaknesses in the $F$ function. This technique applies to a very wide class of round functions.

\section{Slide Attacks on Feistel Ciphers}

In this section, we show how the slide attack can be optimized when it is applied to a Feistel cipher.

KNOWN-PLAINTEXT ATTACKS. In the case of Feistel ciphers, the round function $F((l, r))=(r \oplus f(l), l)$ modifies only half of its input. Therefore, the condition $F(x)=x^{\prime}$ can be recognized by simply comparing the left half of $x$ against the right half of $x^{\prime}$, and this filtering condition eliminates all but $2^{-n / 2}$ of the wrong pairs.

This improved filtering allows us to reduce the time complexity of attack under the known-plaintext threat model to $2^{n / 2}$ known texts and $2^{n / 2}$ offline work. We have a $n$-bit filtering condition on the potential slid pairs, for if $\left(P_{i}, C_{i}\right)$ forms a slid pair with $\left(P_{j}^{\prime}, C_{j}^{\prime}\right)$ then we have $F\left(P_{i}\right)=P_{j}^{\prime}$ and $F\left(C_{i}\right)=C_{j}^{\prime}$. Therefore, potential slid pairs can be identified using a lookup table (or sorted list) with $2^{n / 2}$ entries: we sort the known text $\left(P_{i}, C_{i}\right)$ based on the left halves of $P_{i}$ and $C_{i}$, and for each $j$ we look for a match with the right halves of $P_{j}^{\prime}$ and $C_{j}^{\prime}$.

With this technique, we expect to find one good slid pair along with only one false alarm; false matches can be easily eliminated in a second phase. The slid pair gives us about $n$ bits of information on the key; if this does not reveal all of the key material, we can look for a few more slid pairs or search over the remaining unknown key bits.

Chosen-Plaintext AtTacks. For Feistel ciphers, the data complexity can be reduced further to about $2^{n / 4}$ texts when chosen plaintext queries are available. The key to the reduction in texts is the use of carefully-chosen structures. (This technique was first pioneered by Biham in his work on related-key cryptanalysis [2.) Fix an arbitrary $n / 2$-bit value $x$. We choose a pool of $2^{n / 4}$ plaintexts $P_{i}=\left(x, y_{i}\right)$ by varying over $2^{n / 4}$ random values for $y$, and then choose a second pool of $2^{n / 4}$ texts of the form $P_{j}^{\prime}=\left(y_{j}^{\prime}, x\right)$ by varying over another $2^{n / 4}$ random choices for $y_{j}^{\prime}$. This provides $2^{n / 2}$ pairs of plaintexts, and a right pair occurs with probability $2^{-n / 2}$ (namely, when $f(x)=y_{i} \oplus y_{j}^{\prime}$ ), so we expect to find about one slid pair. This slid pair can be recognized using the $n / 2$-bit filtering condition on the ciphertexts, and then we can use it to recover $n$ bits of key material as before 4 .

Probable-Plaintext ATtacks. When plaintexts contain some redundancy, the data complexity of a known-plaintext slide attack can often be significantly reduced. Our techniques are derived from Biryukov and Kushilevitz's recent work on exploiting such plaintext redundancy in differential attack [4].

\footnotetext{
${ }^{4}$ Notice that if we deal with an unbalanced Feistel cipher, the effect of a chosen plaintext attack can be even greater. For example for a Skipjack-like construction with the same keyed permutation in all rounds, a chosen plaintext attack with only $2^{n / 8}$ time and data is possible.
} 
Consider first a very simple model: the plaintext source emits blocks where the four most significant bits of each byte are always zero, so that the resulting $n$-bit plaintexts have only $n / 2$ bits of entropy. In this case, one can mount a slide attack with about $2^{3 n / 8}$ ciphertexts, which is midway between the data complexities of chosen-plaintext slide attacks $\left(2^{n / 4}\right.$ texts $)$ and known-plaintext slide attacks $\left(2^{n / 2}\right.$ texts, for uniformly-distributed plaintexts).

The observation is that for any fixed value $x$ that can occur as the left half of a plaintext, we expect to see about $2^{3 n / 8-n / 4}=2^{n / 8}$ plaintexts of the form $P_{i}=\left(x, y_{i}\right)$, along with another $2^{n / 8}$ plaintexts of the form $P_{j}^{\prime}=\left(y_{j}^{\prime}, x\right)$. Each $x$ gives about $2^{n / 4}$ pairs of texts, and there are $2^{n / 4}$ values for $x$. Assuming $f$ behaves randomly, any such pair gives a $2^{-n / 2}$ chance of forming a slid pair, so in total we expect to find about one slid pair among all the $2^{3 n / 8}$ ciphertexts.

This attack can even be converted to a ciphertext-only attack with a slight increase in complexity. Suppose the condition $f(u)=v, f\left(u^{\prime}\right)=v^{\prime}$ uniquely identifies the key, and key recovery from $u, u^{\prime}, v, v^{\prime}$ takes $O(1)$ time. Then we can find the key with $2^{3 n / 8+1}$ ciphertexts and $O\left(2^{n / 2}\right)$ work. First, we note that the $n / 2$-bit filtering condition on the ciphertexts gives a set of $2^{n / 4+2}$ potential slid pairs, of which about four are correct (the rest are false matches). The list of potential slid pairs can be identified with $O\left(2^{3 n / 8}\right)$ steps by hashing or sorting. Next, we make a guess at a correct slid pair $C_{i}, C_{j}^{\prime}$. Third, for each remaining potential slid pair $C_{i^{\prime}}, C_{j^{\prime}}^{\prime}$, we compute the key value suggested by the equations $F\left(C_{i}\right)=C_{i^{\prime}}, F\left(C_{j}^{\prime}\right)=C_{j^{\prime}}^{\prime}$, and store this $n$-bit key value in a table. We search for collisions in this table (by hashing or sorting). If our guess at $C_{i}, C_{j}^{\prime}$ indeed gave a correct slid pair, the right key value will be suggested three times. On the other hand, the birthday paradox assures us that wrong key values will be suggested only with negligible probability. This yields an attack that takes $O\left(2^{n / 2}\right)$ time $\left(2^{n / 4}\right.$ guesses at $C_{i}, C_{j}^{\prime}$, performing $O\left(2^{n / 4}\right)$ operations per guess to build the table) and needs $2^{n / 4+2}$ space and about $2^{3 n / 8+1}$ ciphertexts.

Of course, this is only an example. The exact complexity of the probableplaintext and ciphertext-only slide attacks can vary widely: some plaintext distributions increase the complexity of slide attacks (or even render them impossible), while others reduce the complexity substantially. In general, the expected number of texts needed to find the first slid pair is approximately $2^{n / 4}\left(\sum_{x} \operatorname{Pr}[r=\right.$ $x] \operatorname{Pr}[l=x])^{-1 / 2}$ (under heuristic assumptions on $f$ ), although the exact details of the attack will depend intimately on the distribution of the plaintexts.

\section{Modified DES Example: 2K-DES}

The following constitutes in our opinion a nice problem for a student cryptocourse or an introductory crypto-textbook. Suppose one proposes to strengthen DES in the following way. One increases the number of rounds from 16 to 64, and extends the number of key-bits from 56 to 96 in the following simple way: given two independent 48-bit keys $K_{1}, K_{2}$ one uses $K_{1}$ in the odd rounds and $K_{2}$ in the even rounds instead of DES subkeys. This version is obviously immune to exhaustive search. The conventional differential and linear attacks probably 
will also fail due to the increased number of rounds. The question is: "Is this cipher more secure than DES?" Below we show two attacks on this cipher which use the symmetry of the key-scheduling algorithm and are independent of the number of rounds.

One very simple way to attack such cipher is as follows. For any known plaintext-ciphertext pair $(P, C)$, decrypt ciphertext $C$ one round under all possible $2^{32}$ outputs from the $f$ function in the last round. For each of the $2^{32}$ resulting texts $C^{\prime}$, request the encryption $P^{\prime}=E_{K}\left(C^{\prime}\right)$. This is equivalent to decryption all way back to the plaintext $P$ and further by one more round to $F^{-1}\left(P, K_{2}\right)=P^{\prime}$. Since $F$ preserves 32 bits of its input, one can check a 32 -bit filtering condition over $P, P^{\prime}$ to eliminate essentially all of the wrong guesses at $C^{\prime}$. When we find a $C^{\prime}, P^{\prime}$ which survives the filtering condition, we can derive $K_{2}$ easily from the equations $F\left(P^{\prime}, K_{2}\right)=P, F\left(C^{\prime}, K_{2}\right)=C$ (here $F$ includes the Feistel swap of the halves). This procedure leaves only the correct value of $K_{2}$ with high probability. Now $K_{1}$ can be found by exhaustive search; or, for a more efficient attack, we can peel off the first round using the known value of $K_{2}$, and repeat the attack once more on the resulting cipher to learn $K_{1}$. This simple attack uses one known-plaintext $(P, C)$ pair, $2^{33}$ adaptive chosen plaintexts and $2^{33}$ time. A similar attack will actually work for any "almost"-symmetric keyscheduling; see also [3] for another example of this type of attack. Notice that if the number of rounds $r$ is odd and key-scheduling is symmetric then double encryption with such a Feistel-cipher becomes an identity permutation.

This attack can be improved using the ideas of the present paper. By applying slide techniques, we can show that this cipher is much weaker than one would expect even when its number of rounds $r$ is arbitrarily large, and that attacks are available even under the more practical known-plaintext threat model. For any fixed value of $K_{1}, K_{2}$ this cipher can be viewed as a cascade of $\frac{r}{2}$ identical fixed permutations. Thus given a pool of $2^{32}$ known plaintexts, one can recover all 96 bits of the secret key just by checking all the possible pairs in about $2^{63} / 64=2^{57}$ naive steps (each step is equivalent to one $2 \mathrm{~K}$-DES encryption operation). Each pair of plaintexts $\left(P, P^{*}\right)$ suggests $2^{16}$ candidates for $K_{1}$ and $2^{16}$ candidates for $K_{2}$ which are immediately checked against a pair of corresponding ciphertexts $\left(C, C^{*}\right)$. Thus on the average after this process we are left with a few candidate 96-bit keys which can be further checked with trial encryption. Using a more sophisticated approach (ruling out many pairs simultaneously) it is possible to reduce the work factor considerably. For each plaintext we guess the left 24 bits of $K_{1}$, which allows us to calculate 16-bits of the S-box output and thus 16-bits of the possible related plaintext and 16-bits of related ciphertext. This gives a 32-bit condition on the possible related plaintext/ciphertext pair; then analyzing the pool of texts will take a total of $2^{24} \times 2^{32} / 64=2^{50}$ steps.

\section{TREYFER}

In this section we apply slide attacks to cryptanalyze TREYFER, a blockcipher/MAC presented at FSE'97 by Gideon Yuval [10] and aimed at smart-card 
applications. It is characterized by a simple, extremely compact design (only 29 bytes of code) and a very large number of rounds (32). We show an attack on TREYFER that is independent of the number of rounds and exploits the simplicity of key-schedule of this cipher. It uses $2^{32}$ known-plaintexts and requires $2^{44}$ time for analysis.

\section{Description of TREYFER}

TREYFER is a 64-bit block cipher/MAC, with a 64-bit key, designed for a very constrained architectures (like a 8051 CPU with 1KB flash EPROM, 64 bytes RAM, 128 bytes EPROM and peak $1 \mathrm{MHz}$ instruction rate). The algorithm is as follows:

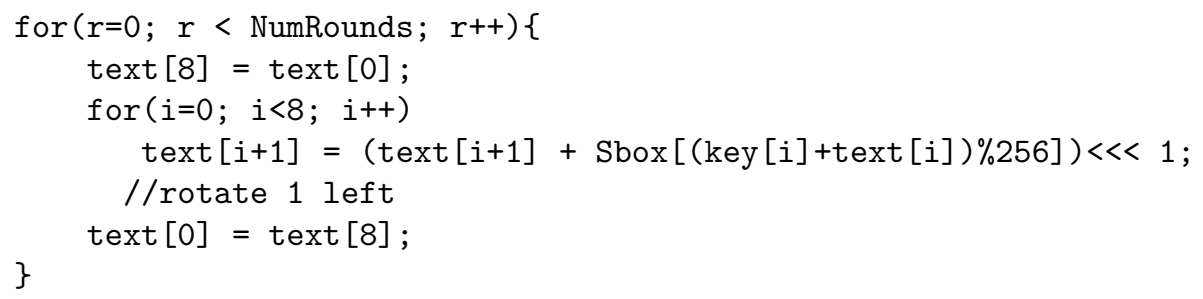

Here text is an eight-byte plaintext, key is an eight-byte key, S-box denotes an 8x8-bit S-box chosen at random, and NumRounds stands for 32 rounds. After 32 rounds of encryption text contains eight-byte ciphertexts. One of the motivations behind the design of this cipher was that in spite of the simplicity of the round function a huge number of rounds (32) will make any possible attack impractical.

As an aside (without any connection to our attack), we observe that TREYFER exhibits much weaker diffusion in the decryption direction: it takes two rounds for a one-byte difference to influence all eight bytes in the encryption direction, but it takes seven rounds in the decryption direction.

\section{Our Attack on TREYFER}

The idea of our attack is very similar to the related-key attacks 212, however our attack is known-plaintext and not chosen-key like the attacks in [2].

In our attack we use the fact that due to hardware constraints the designers of TREYFER sacrificed a proper key-scheduling to make a more compact and faster cipher. Thus key-scheduling of TREYFER simply uses its 64-bit key $K$ byte by byte. This is done exactly in the same fashion at each round.

However the simplicity of key-schedule causes TREYFER to be a cascade of 32 identical permutations! Thus suppose that two plaintexts $P$ and $P^{*}$ are encrypted by TREYFER to $C$ and $C^{*}$. Denote the intermediate encrypted values after each round by $P_{1}, \ldots, P_{32}$, where $P_{32}=C$. Denote the round encryption function of TREYFER by $F$. Now, if two plaintexts are related by a one-round encryption as $F(P, K)=P^{*}$ then it must be that the same relation holds for 
the ciphertexts $F(C, K)=C^{*}$. Due to simplicity of the round function $F$, given a properly related pair the full 64-bit key $K$ of TREYFER can be derived either from equation $F(P, K)=P^{*}$ or from equation $F(C, K)=C^{*}$. If $P, P^{*}$ is a properly related pair both equations suggest the same value of the key. However if the pair is not properly related there is no reason for the two keys to be equal.

Thus on TREYFER with arbitrary number of rounds and with arbitrarily chosen S-box it is possible to mount an attack with about $2^{32}$ known plaintexts and in the time of $2^{44}$ offline TREYFER encryptions (performed on the attacker's computer and not on the slow smart-card processor). Due to the birthday paradox a pool of $2^{32}$ known plaintexts will contain a properly related pair with high probability. Thus a naive approach is to try all the possible $2^{63}$ pairs, and each time the two equations $F(P, K)=P^{*}$ and $F(C, K)=C^{*}$ suggest the same 64-bit key, check this candidate key with trial encryption. Since for each pair we perform 1/16 of the TREYFER encryption, the overall complexity of this naive attack is $2^{59}$ TREYFER encryptions, which is still faster than exhaustive search. However we can do better than that if for each plaintext we do $2^{16}=2^{8} \cdot 2^{8}$ guesses of the two subkeys $\mathrm{k}[7], \mathrm{k}[0]$. For each guess we arrive at a 32-bit condition on the possible co-related plaintext. Thus on the average only one out of $2^{32}$ plaintexts passes the 32-bit condition and it can be easily found in a sorted array of plaintexts. Then the newly formed pair is checked for the version of the full 64-bit key as it was done in a naive approach. The time required by the analysis phase of this attack is equivalent to $2^{16} \cdot 2^{32} \cdot \frac{1}{16}=2^{44}$ TREYFER encryptions.

Thus we have shown an attack on TREYFER, with $2^{32}$ known plaintexts, $2^{44}$ time of analysis and $2^{32}$ memory. The interesting property of this attack is that it is independent of the number of rounds and of the exact choice of the S-box. This attack seems to be on the verge of practicality, due to very slow smart-card encryption (6.4 msec per block) and very slow communication wire (10KBPS) speed. However this task is easily parallelizable if an attacker obtains many smart-cards containing the same secret key. Once the attacker receives the data, the analysis can be done in a few days on an average computer.

It should be possible to make TREYFER immune to this attack by adding a more complex key-schedul€5.

\section{Stream Ciphers and WAKE-ROFB}

It is also possible to mount slide attacks against stream ciphers. We show how to attack the re-synchronization mechanism used in WAKE-ROFB 6 [6], a recent WAKE variant proposed at FSE'98. Our attacks work only under restrictive assumptions on the IV selection and re-synchronization mechanism.

\footnotetext{
${ }^{5}$ Following the results of this paper round counters were introduced into the round function of TREYFER, as a counter-measure against such attacks [11].

${ }^{6}$ WAKE-ROFB is a refinement of a design originally proposed at FSE' 97 [5]. In this paper, we analyze only the FSE'98 scheme; the FSE'97 cipher's re-synchronization mechanism appears to protect it from slide attacks.
} 
Note that this does not reflect poorly on the core of the WAKE-ROFB design; it merely shows that dealing with re-synchronization can be tricky, because it introduces the possibility of chosen-text attacks. (See also 81656].) In short, WAKE-ROFB is not broken. We point out these attacks merely to illustrate the intriguing theoretical possibility of applying slide attacks to stream ciphers.

WAKE-ROFB is a stream cipher with $32 n$ bits of internal state, organized into $n$ 32-bit words. The words are updated via a simple analogue of a non-linear feedback shift register, extended to operate on words instead of bits. Writing $R_{1}, \ldots, R_{n}$ for the state registers, WAKE-ROFB's state update function is defined as

$$
R_{1}^{\prime} \leftarrow R_{n-1}+F\left(R_{n}\right) ; \quad R_{j} \leftarrow R_{j-1} ; \quad R_{1} \leftarrow R_{1}^{\prime}
$$

Here $F: \mathbf{Z}_{2}^{32} \rightarrow \mathbf{Z}_{2}^{32}$ is a bijective key-dependent nonlinear function. Every $k$-th time we step the register, we output the value of $R_{n}$ as the next word of the key-stream. See Figure 3 for a pictorial illustration of the cipher.

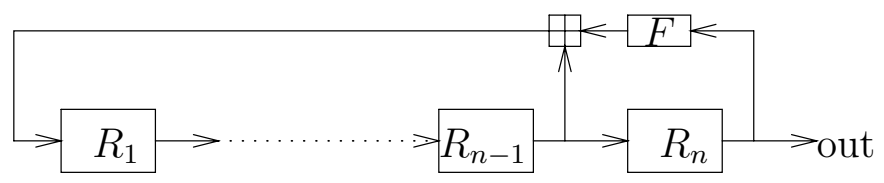

Fig. 3. The WAKE-ROFB stream cipher

The parameters $k$ and $n$ may be varied to suit performance and security needs. However, 6] suggests two concrete proposals: $(n, k)=(5,8)$ and $(n, k)=$ $(4,4)$. For the $n=5$ proposal, a concrete scheme for loading an initialization vector is proposed: the 64 -bit IV $(A, B)$ is loaded into the registers as $R_{1}=R_{4}=$ $R_{5}=A, R_{2}=R_{3}=B$, and then 8 words of output are generated and discarded. For the $n=4$ proposal, no scheme for loading an IV was suggested.

Note that, to support re-synchronization, WAKE-ROFB is built around a mode of operation that is perhaps somewhat unusual for a stream cipher. Many published stream cipher constructions use a public feedback function and load their initial state from the key, and often no allowance is made for re-synchronization. In contrast, WAKE-ROFB is keyed solely by the choice of the keydependent permutation $F$, and the initial state of the register is loaded from a publicly-known IV7. Re-synchronization in WAKE-ROFB is easily accomplished by choosing a new IV.

The main observation is that this construction can be viewed as roughly an unbalanced Feistel cipher (with round function $F$ ) that outputs one word

\footnotetext{
${ }^{7}$ But note that slide attacks do not always require knowledge of the initial state of the register. For instance, some of our attacks would still be possible even if the construction were modified to load the initial state of the register as e.g. the TripleDES-CBC decryption of the IV under some additional keying material.
} 
every $k$ rounds. From this viewpoint, there is no round-dependence in the round transformation. Since Feistel ciphers with no round-dependence are susceptible to slide attacks, it seems natural to suspect that slide attacks may also prove useful against the WAKE-ROFB stream cipher. This is indeed the case.

First, as a warmup exercise, we note that when the attacker has full control over the initial state of the stream cipher, simple slide attacks are often available. The attack is the same as a chosen-plaintext slide attack on a Feistel cipher with constant round subkeys. We fix $r_{1}, \ldots, r_{n-1}$, and generate $2^{16}$ IV's of the form $I V_{X}=\left(r_{1}, \ldots, r_{n-1}, X\right)$ by varying $X$. We also generate $2^{16}$ IV's of the form $I V_{Y}=\left(Y, r_{1}, \ldots, r_{n-1}\right)$ by varying $Y$. Note that if $r_{n-1}+F(X)=Y$, we will have a successful slide relation between the key-stream generated by $I V_{X}$ and the key-stream generated by $I V_{Y}$. For such $X, Y$, the resulting internal states will be closely related: if we let $S_{\alpha}[t]=\left(R_{1, \alpha}[t], \ldots, R_{n, \alpha}[t]\right)$ be the $32 n$-bit state generated from $I V_{\alpha}$ by stepping the cipher $t$ times, then $S_{Y}[t]=S_{X}[t+1]$ for all $t$.

In many cases, this condition can be easily recognized, because the keystreams will be highly related to each other. For instance, for the $(n, k)=(4,4)$ proposal, if we know the key-stream outputs from $I V_{X}$ at times $j k,(j+1) k$ and the key-stream output from $I V_{Y}$ at time $j k$, we can deduce one input-output pair for the $F$ function for each time step; this property allows us to easily recognize slid pairs with about 8 known outputs for the $F$ proposed in $[6]$. Analysis is apparently more difficult when $\operatorname{gcd}(n, k)=1$, but attacks are still available (albeit with increased data requirements) by choosing $n \cdot 2^{32}$ IV's of the form $(Y, \ldots, Y, r, \ldots, r)$; the crucial observation is that $(r, \ldots, r)$ forms a slid pair with $(F(r)+r, r, \ldots, r)$, which forms a slid pair with $(F(r)+r, F(r)+r, r, \ldots, r)$, and so on.

We conclude that a slide attack may be possible with as few as $2^{17}$ streams (each containing at least 8 known outputs), when the attacker has full control over the initial state of the register. This situation might occur if, for instance, the IV-loading mechanism simply loaded the initial state of the register directly as the value of a $n$-word IV, since then an attacker would be able to control the initial state directly with a chosen-IV chosen-ciphertext attack. One corollary is that, to prevent slide attacks, the IV-loading mechanism must be carefully designed. Note that the WAKE-ROFB design precludes these attacks by explicitly specifying a resynchronization mechanism that allows only limited control over the initial state of the cipher.

Even when the attacker has no control over the initial state of the register, known-IV slide attacks may still be possible. By analogy to the standard knowntext attacks on block ciphers, we expect to find one successful slide relation after

8 This is because [6] constructs the $T$ table from two $4 \times 16$-bit lookup tables, and by the birthday paradox after 7 observations of a 4-bit value we expect to see a collision or two. But even for more sophisticated constructions of the $F$ function, the number of known outputs needed would not increase substantially. With a randomly generated $T$ table, about 40 known outputs would suffice; even if the entire function $F$ were chosen randomly, $2^{16.5}-2^{17.5}$ known outputs should be enough to detect slid pairs. 
examining about $2^{32 n / 2}$ known text streams, and in some cases this might enable successful cryptanalysis of the cipher. One simple defense is to increase the size of the internal state enough so that the data requirements become infeasible.

Finally, we consider the concrete IV-loading scheme proposed in [6] for the $(n, k)=(5,8)$ WAKE-ROFB cipher. There the 64-bit IV $(A, B)$ is loaded into the registers as $\left(R_{1}, \ldots, R_{5}\right)=(A, B, B, A, A)$, and then 8 words of output are generated and discarded.

We note that a slide attack on this scheme is still possible, when $2^{32}$ chosenIV queries are available. We obtain known key-stream output for the $2^{32}$ IV's of the form $(A, A)$. This loads the initial state of the registers with $\left(R_{1}, \ldots, R_{5}\right)=$ $(A, \ldots, A)$. Note that when $F(A)=0$, we will have $R_{1}^{\prime}=A$, and so stepping the initial state $(A, \ldots, A)$ gives the state $(A, \ldots, A)$. In other words, for $A=$ $F^{-1}(0)$, we obtain a cycle of period one. This can be easily recognized from a short stretch of known key-stream output, and allows allows us to obtain 32 bits of information on the key.

It is clear that the design of a secure IV-loading mechanism for WAKEROFB-like stream ciphers is non-trivial. Certainly running the cipher for $8 k$ time steps and discarding the outputs helps stop some attacks, but as we have shown, it is not always sufficient.

Therefore, we propose the following design principle for such stream cipher\$:

Whenever possible, the feedback function should include some form of round-dependence.

\section{Key-Dependent S-Boxes: A Variant of Blowfish}

The following was inspired by a paper due to Grossman and Tuckerman 9 from 1978. In this section we show by using a more modern techniques that if the only strength of a cipher comes from key-dependent S-boxes (with no round dependence) then such cipher can be attacked easily using slide attacks. This shows that slide attacks are not restricted to ciphers with weak key-scheduling algorithms.

For an example of how this might work consider a cipher called Blowfish, which was designed by Bruce Schneier [14]. This is a Feistel cipher with 64-bit block, 16 rounds and up to 448 bits of the secret key. These are expanded into a table consisting of four S-boxes from 8 to 32 bits (4096 bytes total). S-boxes are key-dependent and unknown to the attacker. Also in each round a 32-bit subkey $P_{i}$ is xored to one of the inputs. At the end two 32-bit subkeys $P_{17}$ and $P_{18}$ are xoRed to the output of a cipher. See Figure 4 for a picture of one round of Blowfish. So far no attacks are known on a full version of this cipher.

\footnotetext{
${ }^{9}$ Following the results of this paper, round counters were introduced into the resynchronization mechanism of WAKE-ROFB as a counter-measure against such attacks 7 .
} 


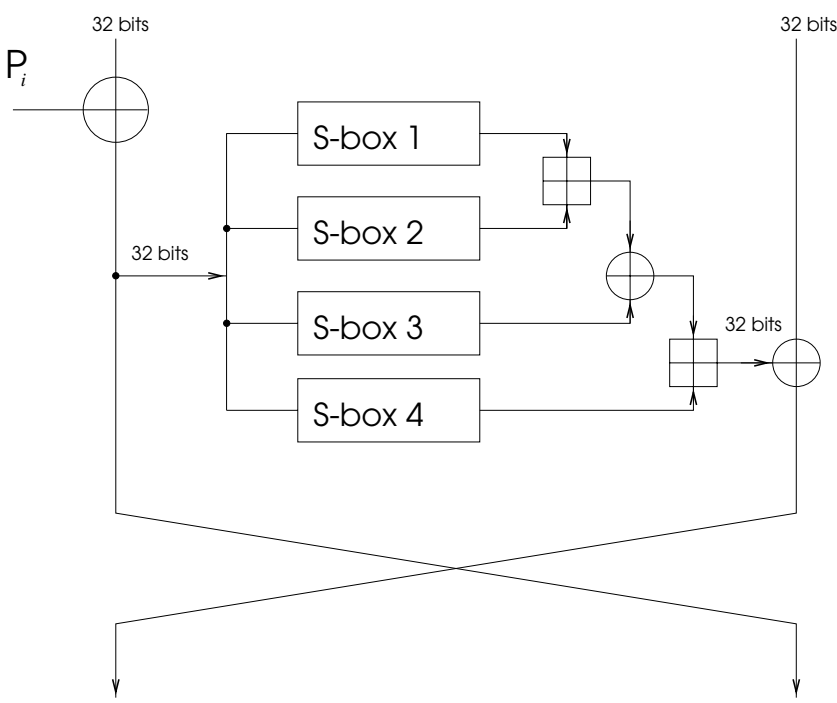

Fig. 4. One round of Blowfish.

The best previous result [15] is a differential attack on Blowfish with known S-boxes which can find the $P_{i}$ array using $2^{8 r+1}$ chosen plaintexts, where $r$ stands for the number of rounds. For certain weak keys that generate bad S-boxes (1 out $2^{14}$ keys) the same attack requires $2^{4 r+1}$ chosen plaintexts (still completely ineffective against 16-round Blowfish).

Assume that all the $P_{i}$ 's are equal to zero. In this case one may notice that all rounds of a cipher perform the same transformation which is data-dependent. Thus given a 32-bit input to the $F$-function the output of the $F$ function is uniquely determined. Also only 16 bytes out of 4096 take part in each evaluation of the $F$-function. Thus one naive approach will be to fix a plaintext $P$, guess all these 128-bits of the key and partially encrypt $P$ with the guessed keys one Feistel-round, and then perform a slide attack for $P$ and for the guessed text. A less naive approach is to guess the 32-bit output of the $F$-function and thus obtain a correct encryption with one Feistel round in $2^{32}$ steps, checking if the guess was correct with a usual sliding technique. An even better approach is to encrypt two pools of chosen plaintexts $\left(X, P_{R}\right)$ and $\left(P_{R}, Y\right)$, where $X$ and $Y$ both receive $2^{16}$ random values and $P_{R}$ is fixed. Thus with high probability there is an element $\left(P_{R}, Y_{i}\right)$ in the second pool which is an exact one-round encryption of some element $\left(X_{j}, P_{R}\right)$ from the first pool. Such pair can be easily detected by sliding considerations (especially if we repeat this experiment with the same value of $P_{R}$ and other random values of $X$ and $Y$ ).

Each slid pair provides us with about 64 bits of key-dependent S-box information (two equations for $F$-function). Thus with about 500 probes of this type it is possible to find all four S-boxes. Data for this attack can be packed into structures efficiently. Thus we have a very simple slide attack with only about 
$2^{9} \cdot 2^{18}=2^{27}$ chosen plaintexts on this variant of Blowfish. Similar techniques can be applied to any iterative autokey cipher.

This attack is independent of the number of rounds of a cipher (be it 16 or 16000 rounds), of the exact structure of the $F$-function, of the key length, and of

the key-schedule, no matter how complex is the S-box generation process. This shows that slide attacks are not restricted to ciphers with weak key-scheduling.

\section{Acknowledgments}

Many thanks to Bruce Schneier for suggesting the name "slide attack", and for encouragement to write up these results. Also, we are grateful to Craig Clapp, Lars Knudsen, and the FSE6 program committee for detailed comments on the paper.

\section{References}

1. E. Biham, A. Shamir, Differential Cryptanalysis of the Data Encryption Standard, Springer-Verlag, 1993.

2. E. Biham, New Types of Cryptanalytic Attacks Using Related Keys, J. of Cryptology, Vol.7, pp.229-246, 1994.

3. E. Biham, A. Biryukov, N. Ferguson, L. R. Knudsen, B. Schneier, A. Shamir, Cryptanalysis of Magenta, Second AES Conference, 1999.

4. A. Biryukov, E. Kushilevitz, From differential cryptanalysis to ciphertext-only attacks, LNCS 1462, Advances in Cryptology-CRYPTO'98, pp.72-88, SpringerVerlag, 1998.

5. C. Clapp, Optimizing a Fast Stream Cipher for VLIW, SIMD, and Superscalar Processors, LNCS 1267, FSE'97 Proceedings, pp.273-287, Springer-Verlag, 1997.

6. C. Clapp, Joint Hardware / Software Design of a Fast Stream Cipher, LNCS 1373, FSE'98 Proceedings, pp.75-92, Springer-Verlag, 1998.

7. C. Clapp, WAKE UPdate, FSE'99 rump session, 1999.

8. J. Daemen, R. Govaerts, J. Vanderwalle, Re-synchronization weaknesses in stream ciphers, LNCS 765, Advances in Cryptology-EUROCRYPT'93, pp.159-169, Springer-Verlag, 1994.

9. E. K. Grossman, B. Tuckerman, Analysis of a Weakened Feistel-like Cipher, 1978 International Conference on Communications, pp.46.3.1-46.3.5, Alger Press Limited, 1978.

10. G. Yuval, Reinventing the Travois: Encryption/MAC in 30 ROM Bytes, LNCS 1267, FSE'97 Proceedings, pp.205-209, Springer-Verlag, 1997.

11. G. Yuval, Private communication, August 1998.

12. L. R. Knudsen, Cryptanalysis of LOKI91, LNCS 718, Advances in CryptologyAUSCRYPT'92, pp.196-208, Springer-Verlag, 1993.

13. M. Matsui, Linear Cryptanalysis Method of DES Cipher, LNCS 765, Advances in Cryptology-EUROCRYPT'93, pp.386-397, Springer-Verlag, 1994.

14. B. Schneier, Description of a New Variable-Length Key, 64-Bit Block Cipher (Blowfish), LNCS 809, FSE'94 Proceedings, pp.191-204, Springer-Verlag, 1994.

$\overline{10}$ Notice also, that it is possible to find a 448-bit key which will force $P_{1}, \ldots, P_{14}$ to be zero; however, $P_{15}, . ., P_{18}$ will remain uncontrolled. 
15. S. Vaudenay, On the Weak Keys in Blowfish, LNCS 1039, FSE'96 Proceedings, pp.27-32, Springer-Verlag, 1996.

16. D. Wagner, Cryptanalysis of some recently-proposed multiple modes of operation, LNCS 1373, FSE'98 Proceedings, pp.254-269, Springer-Verlag, 1998. 